\title{
Sign-changing solutions to discrete fourth-order Neumann boundary value problems
}

Jingping Yang*

\section{"Correspondence: fuj09@|zu.cn Gansu Institute of Political Science and Law, Lanzhou, 730070,} P.R. China

\begin{abstract}
By using topological degree theory and fixed point index theory, we consider a discrete fourth-order Neumann boundary value problem. We provide sufficient conditions for the existence of sign-changing solutions, positive solutions, and negative solutions.
\end{abstract}

Keywords: topological degree theory; fixed point index theory; sign-changing solutions; positive solutions; negative solutions

\section{Introduction}

In this paper, we consider the existence of sign-changing solutions to the following discrete nonlinear fourth-order boundary value problem (BVP):

$$
\begin{aligned}
& \Delta^{4} u(t-2)-\alpha \Delta^{2} u(t-1)+\beta u(t)=f(t, u(t)), \quad t \in[2, T]_{\mathbb{Z}}, \\
& \Delta u(1)=\Delta u(T)=\Delta^{3} u(0)=\Delta^{3} u(T-1)=0,
\end{aligned}
$$

where $\Delta$ denotes the forward difference operator defined by $\Delta u(t)=u(t+1)-u(t), T>2$ is an integer, $f:[2, T]_{\mathbb{Z}} \times \mathbb{R} \rightarrow \mathbb{R}$ is continuous, and $\alpha, \beta$ are real parameters and satisfy

$$
\alpha^{2} \geq 4 \beta, \quad \text { and } \quad \alpha-\sqrt{\alpha^{2}-4 \beta}>-8 \sin ^{2} \frac{\pi}{2(T-1)} .
$$

Let $a, b$ be two integers with $a<b$. We employ $[a, b]_{\mathbb{Z}}$ to denote the discrete interval given by $\{a, a+1, \ldots, b\}$.

The theory of nonlinear difference equations has been widely used to study discrete models in many fields such as computer science, economics, neural network, ecology, cybernetics, etc. In recent years, a great deal of work has been done in the study of the existence and multiplicity of solutions for a discrete boundary value problem. For the background and recent results, we refer the reader to [1-5] and the references therein.

We may think of BVP (1.1), (1.2) as a discrete analogue of the fourth-order boundary value problem

$$
\begin{aligned}
& u^{(4)}(t)-\alpha u^{(2)}(t)+\beta u(t)=\lambda f(t, u(t)), \quad t \in(0,1), \\
& u^{\prime}(0)=u^{\prime}(1)=u^{3}(0)=u^{3}(1)=0 .
\end{aligned}
$$

( 2013 Yang; licensee Springer. This is an Open Access article distributed under the terms of the Creative Commons Attribution License (http://creativecommons.org/licenses/by/2.0), which permits unrestricted use, distribution, and reproduction in any medium, provided the original work is properly cited. 
The special case of BVP (1.3), (1.4) has been studied by many authors using various approaches; for example, see [6,7].

However, it seems that there is no similar result in the literature on the existence of sign-changing solutions, positive solutions, and negative solutions for BVP (1.1), (1.2). Motivated by [8], our purpose is to apply some basic theorems in topological degree theory and fixed point index theory to establish some conditions for the nonlinear function $f$, which are able to guarantee the existence of sign-changing solutions, positive solutions, and negative solutions for the above discrete boundary value problem.

The organization of this paper is as follows. In Section 2, we state some notations and preliminary knowledge about the topological degree theory and fixed point index theory. In Section 3, we present the spectrum of second-order eigenvalue problems. In Section 4, we give the expression of Green's function of second-order Neumann problems and consider the eigenvalue problem of fourth-order BVPs. In Section 5, by computing the topological degree and the fixed point index, we discuss the existence of multiple sign-changing solutions to BVP (1.1), (1.2).

\section{Preliminaries}

As we have mentioned, we will use the theory of the Leray-Schauder degree and the fixed point index in a cone to prove our main existence results. Let us collect some results that will be used below. One can refer to [9-12] for more details.

Lemma 2.1 (see $[9,10])$ Let $E$ be a Banach space and $X \subset E$ be a cone in E. Assume that $\Omega$ is a bounded open subset of $E$. Suppose that $A: X \cap \bar{\Omega} \rightarrow X$ is a completely continuous operator. If there exists $x_{0} \in X \backslash\{\theta\}$ such that

$$
x-A x \neq \mu x_{0}, \quad \forall x \in X \cap \partial \Omega, \mu \geq 0,
$$

then the fixed point index $i(A, X \cap \Omega, X)=0$.

Lemma 2.2 (see $[9,10])$ Let $E$ be a Banach space and let $X \subset E$ be a cone in E. Assume that $\Omega$ is a bounded open subset of $E, \theta \in \Omega$. Suppose that $A: X \cap \bar{\Omega} \rightarrow X$ is a completely continuous operator. If

$$
A x \neq \mu x, \quad \forall x \in X \cap \partial \Omega, \mu \geq 1,
$$

then the fixed point index $i(A, X \cap \Omega, X)=1$.

Lemma 2.3 (see [11]) Let E be a Banach space, let $\Omega$ be a bounded open subset of $E, \theta \in \Omega$, and $A: \bar{\Omega} \rightarrow E$ be completely continuous. Suppose that

$$
\|A x\| \leq\|x\|, \quad A x \neq x, \forall x \in \partial \Omega
$$

Then $\operatorname{deg}(I-A, \Omega, \theta)=1$.

Lemma 2.4 (see [12]) Let $A$ be a completely continuous operator which is defined on a Banach space E. Let $x_{0} \in E$ be a fixed point of $A$ and assume that $A$ is defined in a neighborhood of $x_{0}$ and Fréchet differentiable at $x_{0}$. If 1 is not an eigenvalue of the linear operator 
$A^{\prime}\left(x_{0}\right)$, then $x_{0}$ is an isolated singular point of the completely continuous vector field I-A, and for small enough $r>0$,

$$
\operatorname{deg}\left(I-A, B\left(x_{0}, r\right), \theta\right)=(-1)^{k},
$$

where $k$ is the sum of algebraic multiplicities of real eigenvalues of $A^{\prime}\left(x_{0}\right)$ in $(1,+\infty)$.

Lemma 2.5 (see [12]) Let A be a completely continuous operator which is defined on a Banach space E. Assume that 1 is not an eigenvalue of the asymptotic derivative. Then the completely continuous vector field $I-A$ is nonsingular on spheres $S_{\rho}=\{x \in E:\|x\|=\rho\}$ of sufficiently large radius $\rho$ and

$$
\operatorname{deg}(I-A, B(\theta, \rho), \theta)=(-1)^{k},
$$

where $k$ is the sum of algebraic multiplicities of real eigenvalues of $A^{\prime}(\infty)$ in $(1,+\infty)$.

Lemma 2.6 (see [12]) Let $X$ be a solid cone of a Banach space $E$ ( $X^{\circ}$ is nonempty), let $\Omega$ be a relatively bounded open subset of $X$, and let $A: X \rightarrow X$ be a completely continuous operator. If any fixed point of $A$ in $\Omega$ is an interior point of $X$, there exists an open subset $O$ of $E(O \subset \Omega)$ such that

$$
\operatorname{deg}(I-A, O, \theta)=i(A, \Omega, X) .
$$

Now, we will consider the space

$$
E=\left\{u:[2, T]_{\mathbb{Z}} \rightarrow \mathbb{R}, \Delta u(1)=\Delta u(T)=\Delta^{3} u(0)=\Delta^{3} u(T-1)=0\right\}
$$

equipped with the norm $\|u\|=\max _{t \in[2, T]_{\mathbb{Z}}}|u(t)|$. Clearly, $E$ is a $(T-1)$-dimensional Banach space. Choose the cone $P \subset E$ defined by

$$
P=\left\{u \in E \mid u(t) \geq 0, t \in[2, T]_{\mathbb{Z}}\right\}
$$

Obviously, the interior of $P$ is $P^{\circ}=\left\{u \in E \mid u(t)>0, t \in[2, T]_{\mathbb{Z}}\right\}$. For each $u, v \in E$, we write $u \geq v$ if $u(t) \geq v(t)$ for $t \in[2, T]_{\mathbb{Z}}$. A solution $u$ of BVP (1.1), (1.2) is said to be a positive solution (a negative solution, resp.) if $u \in P \backslash\{\theta\}(u \in(-P) \backslash\{\theta\}$, resp.). A solution $u$ of BVP (1.1), (1.2) is said to be a sign-changing solution if $u \notin P \cup(-P)$.

\section{Spectrum of second-order eigenvalue problems}

Consider the second-order discrete linear eigenvalue problems

$$
\begin{aligned}
& -\Delta^{2} y(t-1)+\lambda y(t)=0, \quad t \in[1, T]_{\mathbb{Z}}, \\
& \Delta y(1)=\Delta y(T)=0 .
\end{aligned}
$$

Lemma 3.1 (see [13] Theorem 1) The eigenvalues of (3.1), (3.2) can be given by

$$
\lambda_{k}=2 \cos \frac{k \pi}{T+1}-2,
$$


and the corresponding eigenfunction is if $k=0$, then $\varphi_{0}(t)=1$. If $k \in\{1,2, \ldots, T-2\}$, we have

(i) if $k \neq \frac{T-1}{3}$, then $\varphi_{k}(t)=\frac{1}{\cos \frac{3 k \pi}{2(T-1)}} \cos \frac{k \pi}{(2 T-1)}(2 t-3)$,

(ii) if $k=\frac{T-1}{3}$, then $\varphi_{k}(t)=\sin \frac{\pi t}{3}$.

\section{Green's function and eigenvalue problems to fourth-order BVPs}

In this section, we construct Green's function associated with BVP (1.1), (1.2).

Let $r_{1}, r_{2}$ be roots of the polynomial $P(r)=r^{2}-\alpha r+\beta$, namely,

$$
r_{1}=\frac{\alpha+\sqrt{\alpha^{2}-4 \beta}}{2}, \quad r_{2}=\frac{\alpha-\sqrt{\alpha^{2}-4 \beta}}{2} .
$$

Then we have

$$
\begin{aligned}
\Delta^{4} u(t-2)-\alpha \Delta^{2} u(t-1)+\beta u(t) & =\left(-\Delta^{2} L+r_{1}\right)\left(-\Delta^{2} L+r_{2}\right) u(t) \\
& =\left(-\Delta^{2} L+r_{2}\right)\left(-\Delta^{2} L+r_{1}\right) u(t),
\end{aligned}
$$

where $u=(u(0), u(1), \ldots, u(T+2)), L u=u(t-1), t \in[2, T]_{\mathbb{Z}}$. Under the basic assumption on $\alpha, \beta$, it is easy to see that $r_{1} \geq r_{2}>-4 \sin ^{2} \frac{\pi}{2(T-1)}$.

Consider the two initial value problems:

$$
\begin{aligned}
& \left\{\begin{array}{l}
-\Delta^{2} u(t-1)+r_{i} u(t)=0, \\
\Delta u(1)=0, \quad u(1)=1,
\end{array}\right. \\
& \left\{\begin{array}{l}
-\Delta^{2} v(t-1)+r_{i} v(t)=0, \\
\Delta v(T)=0, \quad v(T)=1 .
\end{array}\right.
\end{aligned}
$$

By the direct computing, we get (4.1) has a unique solution

$$
u(t)=\frac{r_{2}-1}{r_{1}\left(r_{2}-r_{1}\right)} r_{1}^{t}+\frac{1-r_{1}}{r_{2}\left(r_{2}-r_{1}\right)} r_{2}^{t},
$$

and (4.2) has a unique solution

$$
v(t)=\frac{r_{2}-1}{r_{1}^{T}\left(r_{2}-r_{1}\right)} r_{1}^{t}+\frac{1-r_{1}}{r_{2}^{T}\left(r_{2}-r_{1}\right)} r_{2}^{t} .
$$

Let

$$
\rho:=\frac{\left(r_{1}-1\right)\left(r_{2}-1\right)\left(r_{2}^{T-1}-r_{1}^{T-1}\right)}{r_{1}^{T-1} r_{2}^{T-1}} .
$$

Lemma 4.1 Let $h:[1, T]_{\mathbb{Z}} \rightarrow \mathbb{R}$ and $i \in\{1,2\}$ be fixed. Then the problem

$$
\left\{\begin{array}{l}
-\Delta^{2} u(t-1)+r_{i} u(t)=h(t), \quad t \in[2, T]_{\mathbb{Z}} \\
\Delta u(1)=\Delta u(T)=0
\end{array}\right.
$$

has a unique solution

$$
u(t)=\sum_{s=2}^{T} G_{i}(t, s) h(s), \quad t \in[1, T+1]_{\mathbb{Z}},
$$


where $G_{i}(t, s)$ is given by

$$
G_{i}(t, s)=\frac{1}{\rho} \begin{cases}u(t) v(s), & 1 \leq s \leq t \leq T+1 \\ u(s) v(t), & 1 \leq t \leq s \leq T+1\end{cases}
$$

Remark 4.1 Green's function $G_{i}(t, s)$ defined by Lemma 4.1 is positive on $[1, T]_{\mathbb{Z}} \times[1, T]_{\mathbb{Z}}$.

Define operators $K, f, A: E \rightarrow E$, respectively, by

$$
\begin{aligned}
& (K u)(t)=\sum_{s=2}^{T} \sum_{k=2}^{T} G_{2}(t, k) G_{1}(k, s) u(s), \quad u \in E, t \in[1, T]_{\mathbb{Z}} \\
& (f u)(t)=f(t, u(t)), \quad u \in E, t \in[1, T]_{\mathbb{Z}} ; \\
& A=K f .
\end{aligned}
$$

Now, from Lemma 4.1, it is easy to see that BVP (1.1), (1.2) has a solution $u=u(t)$ if and only if $u$ is a fixed point of the operator $A$. It follows from the continuity of $f$ that $A: E \rightarrow E$ is completely continuous.

Lemma 4.2 Let $h:[2, T]_{\mathbb{Z}} \rightarrow R$. Then the linear discrete fourth-order boundary value problem

$$
\left\{\begin{array}{l}
\Delta^{4} u(t-2)-\alpha \Delta^{2} u(t-1)+\beta u(t)=h(t), \quad t \in[2, T]_{\mathbb{Z}} \\
\Delta u(1)=\Delta u(T)=\Delta^{3} u(0)=\Delta^{3} u(T-1)=0
\end{array}\right.
$$

has a unique solution

$$
\begin{aligned}
u(t) & =\sum_{s=2}^{T}\left(\sum_{k=2}^{T} G_{1}(t, k) G_{2}(k, s) h(s)\right) \\
& =\sum_{s=2}^{T}\left(\sum_{k=2}^{T} G_{2}(t, k) G_{1}(k, s) h(s)\right), \quad t \in[2, T]_{\mathbb{Z}},
\end{aligned}
$$

and

$$
u(0)=u(3), \quad u(1)=u(2), \quad u(T)=u(T+1), \quad u(T-1)=u(T+2) .
$$

Proof The conclusion is obvious, so we omit it.

We will use the following assumptions.

(H1) $\alpha, \beta$ are real parameters and satisfy

$$
\alpha^{2} \geq 4 \beta, \quad \text { and } \quad \alpha-\sqrt{\alpha^{2}-4 \beta}>-8 \sin ^{2} \frac{\pi}{2(T-1)} .
$$

(H2) $f:[2, T]_{\mathbb{Z}} \times \mathbb{R} \rightarrow \mathbb{R}$ is continuous, for any $t \in[2, T]_{\mathbb{Z}}, f(t, 0)=0$; for any $t \in[2, T]_{\mathbb{Z}}$ and $x \in \mathbb{R}, x f(t, x) \geq 0$. 
(H3) There exists an even number $k_{0} \in[0, T-2]_{\mathbb{Z}}$ such that

$$
\frac{1}{\lambda_{k_{0}}^{2}}<\beta_{0}<\frac{1}{\lambda_{k_{0}+1}^{2}},
$$

where $\lim _{x \rightarrow 0} \frac{f(t, x)}{x}=\beta_{0}$ uniformly for $t \in[2, T]_{\mathbb{Z}}, \lambda_{k}$ is defined in Lemma 3.1 and $\lambda_{T-1} \triangleq \infty$.

(H4) There exists an even number $k_{1} \in[0, T-2]_{\mathbb{Z}}$ such that

$$
\frac{1}{\lambda_{k_{1}}^{2}}<\beta_{\infty}<\frac{1}{\lambda_{k_{1}+1}^{2}}
$$

where $\lim _{x \rightarrow \infty} \frac{f(t, x)}{x}=\beta_{\infty}$ uniformly for $t \in[2, T]_{\mathbb{Z}}$, and $\lambda_{0}, \lambda_{1}, \ldots, \lambda_{T-1}$ are given in the condition (H3).

(H5) There exists a constant $M>0$ such that for any $(t, x) \in[2, T]_{\mathbb{Z}} \times[-M, M]$,

$$
|f(t, x)|<\omega^{-1} M
$$

where $\omega=\max _{t \in[2, T]_{\mathbb{Z}}} \sum_{s=2}^{T} \sum_{k=2}^{T} G_{2}(t, k) G_{1}(k, s)$.

Lemma 4.3 Suppose that (H2) holds and $u \in P \backslash\{\theta\}$ is a solution of BVP (1.1), (1.2). Then $u \in P^{\circ}$.

Proof According to (H2) and the positivity of Green's function defined in Lemma 4.1, we can easily get the desired conclusion.

Remark 4.2 Similarly to Lemma 4.3, we also know that if (H2) holds and $u \in(-P) \backslash\{\theta\}$ is a solution of BVP (1.1), (1.2), then $u \in(-P)^{\circ}$.

Lemma 4.4 Suppose that (H2)-(H4) hold. Then the operator $A$ is Fréchet differentiable at $\theta$ and $\infty$, where the operator $A$ is defined by (4.5). Moreover, $A^{\prime}(\theta)=\beta_{0} K$ and $A^{\prime}(\infty)=$ $\beta_{\infty} K$.

Proof By (H3), for any $\epsilon>0$, there exists $\delta>0$ such that $\left|f(t, x)-\beta_{0} x\right|<\epsilon|x|$ for any $0<$ $|x|<\delta, t \in[2, T]_{\mathbb{Z}}$. Hence, noticing that $f(t, 0)=0$ for any $t \in[2, T]_{\mathbb{Z}}$, we have

$$
\begin{aligned}
\left\|A u-A \theta-\beta_{0} K u\right\| & =\left\|K\left(f u-\beta_{0} u\right)\right\| \\
& \leq\|K\| \max _{t \in[2, T]_{\mathbb{Z}}}\left|f(t, u(t))-\beta_{0} u(t)\right|<\epsilon\|K\|\|u\|
\end{aligned}
$$

for any $u \in E$ with $0<|u|<\delta$, where $\|K\|=\max _{t \in[2, T]_{\mathbb{Z}}} \sum_{s=2}^{T} \sum_{k=2}^{T}\left|G_{2}(t, k)\right|\left|G_{1}(k, s)\right|$. Consequently,

$$
\lim _{\|u\| \rightarrow 0} \frac{\left\|A u-A \theta-\beta_{0} K u\right\|}{\|u\|}=0 .
$$

This means that the nonlinear operator $A$ is Fréchet differentiable at $\theta$ and $A^{\prime}(\theta)=\beta_{0} K$.

By (H4), for any $\epsilon>0$, there exists $W>0$ such that $\left|f(t, x)-\beta_{\infty} x\right|<\epsilon|x|$ for any $|x|>$ $W, t \in[2, T]_{\mathbb{Z}}$. Let $c=\max _{(t, x) \in[2, T]_{\mathbb{Z}} \times[-W, W]}\left|f(t, x)-\beta_{\infty} x\right|$. By the continuity of $f(t, x)$ with 
respect to $x$, we have $c<+\infty$. Then, for any $(t, x) \in[2, T]_{\mathbb{Z}} \times \mathbb{R},\left|f(t, x)-\beta_{\infty} x\right|<\epsilon|x|+c$. Thus

$$
\left\|A u-\beta_{\infty} K u\right\| \leq\|K\| \cdot \max _{t \in[2, T]_{\mathbb{Z}}}\left|f(t, u)-\beta_{\infty} u(t)\right|<\|K\|(\epsilon\|u\|+c)
$$

for any $u \in E$. Consequently,

$$
\lim _{\|u\| \rightarrow \infty} \frac{\left\|A u-A \theta-\beta_{\infty} K u\right\|}{\|u\|}=0,
$$

which implies that operator $A$ is Fréchet differentiable at $\infty$ and $A^{\prime}(\infty)=\beta_{\infty} K$. The proof is completed.

Lemma 4.5 Let $M$ be given in the condition (H5). Suppose that (H1)-(H4) hold. Then $A(P) \subset P, A(-P) \subset(-P)$. Moreover, one has the following.

(i) There exists an $r_{0} \in(0, M)$ such that for any $0<r \leq r_{0}$,

$$
i(A, P \cap B(\theta, r), P)=0, \quad i(A,(-P) \cap B(\theta, r),-P)=0 .
$$

(ii) There exists an $R_{0}>M$ such that for any $R \geq R_{0}$,

$$
i(A, P \cap B(\theta, R), P)=0, \quad i(A,(-P) \cap B(\theta, R),-P)=0 .
$$

Proof By $(\mathrm{H} 2)$ and the fact that $G_{i}(t, s)$ is positive on $[2, T]_{\mathbb{Z}} \times[2, T]_{\mathbb{Z}}$, we get that for any $t \in[2, T]_{\mathbb{Z}}, f(t, P) \subset P, f(t,-P) \subset-P$, and $K(P) \subset P, K(-P) \subset-P$. Then $A(P) \subset P$ and $A(-P) \subset-P$.

We only need to prove conclusion (i). The proof of conclusion (ii) is similar and will be omitted here. Let $\gamma_{0}=\inf _{\|u\|=1}\left\|u-\beta_{0} K u\right\|$. The condition (H3) yields $\gamma_{0}>0$. It follows from (4.12) that there exists $r_{0} \in[0, M]$ such that

$$
\left\|A u-\beta_{\infty} K u\right\|<\frac{1}{2} \gamma_{0}\|u\|,
$$

where $0<u \leq r_{0}$. Setting $H(s, u)=s A u+(1-s) \beta_{0} K u$, then $H:[0,1] \times E \rightarrow E$ is completely continuous. For any $s \in[0,1]$ and $0<u \leq r_{0}$, we obtain that

$$
\|u-H(s, u)\| \geq\left\|u-\beta_{0} K u\right\|-s\left\|A u-\beta_{0} K u\right\| \geq \gamma_{0}\|u\|-\frac{1}{2} \gamma_{0}\|u\|>0 .
$$

According to the homotopy invariance of the fixed point index, for any $0<r \leq r_{0}$, we have

$$
\begin{aligned}
& i(A, P \cap B(\theta, r), P)=i\left(\beta_{0} K, P \cap B(\theta, r), P\right), \\
& i(A,-P \cap B(\theta, r),-P)=i\left(\beta_{0} K,-P \cap B(\theta, r),-P\right) .
\end{aligned}
$$

Let $\varphi_{0}(t)=1$. Then $K \varphi_{0}=\lambda_{0}^{2} \varphi_{0}$ and $\varphi_{0} \in P$ (see Lemma 3.1 and the proof of Lemma 4.2). We claim

$$
u-\beta_{0} K u \neq \sigma \varphi_{0}, \quad \forall u \in P \cap \partial B(\theta, r), \sigma \geq 0 .
$$


Indeed, we assume that there exist $u_{1} \in P \cap \partial B(\theta, r)$ and $\sigma_{1} \geq 0$ such that $u_{1}-\beta_{0} K u_{1}=\sigma_{1} \varphi_{0}$. Obviously, $u_{1}=\beta_{0} K u_{1}+\sigma_{1} \varphi_{0} \geq \sigma_{1} \varphi_{0}$. Since $\beta_{0} \neq \lambda_{k}^{-2}, k=1,2, \ldots, T-2$, then $\sigma_{1}>0$. Set $\sigma_{\max }=\sup \left\{\sigma: u_{1} \geq \sigma \varphi_{0}\right\}$. It is clear that $\sigma_{1} \leq \sigma_{\max }<\infty$ and $u_{1} \geq \sigma_{\max } \varphi_{0}$. Then

$$
u_{1}=\beta_{0} K u_{1}+\sigma_{1} \varphi_{0} \geq \beta_{0} K \sigma_{\max } \varphi_{0}+\varphi_{0} \sigma_{1}=\left(\beta_{0} \lambda_{0}^{2} \sigma_{\max }+\sigma_{1}\right) \varphi_{0} .
$$

Since $\beta_{0} \lambda_{0}^{2}>1$, then $\beta_{0} \lambda_{0}^{2} \sigma_{\max }+\sigma_{1}>\sigma_{\max }$, which contradicts the definition of $\sigma_{\max }$. This proves (4.21).

It follows from Lemma 2.1 and (4.20) that

$$
i\left(\beta_{0} K, P \cap B(\theta, r), P\right)=0 .
$$

Similarly to (4.22), we know also that

$$
i\left(\beta_{0} K,-P \cap B(\theta, r),-P\right)=0 .
$$

By (4.18), (4.19), (4.22), and (4.23), we conclude

$$
i(A, P \cap B(\theta, r), P)=0, \quad i(A,-P \cap B(\theta, r),-P)=0 .
$$

\section{Main results}

Now, with the aid of the lemmas in Section 2, we are in a position to state and prove our main results.

Theorem 5.1 Assume that the conditions (H1)-(H5) hold. Then BVP (1.1), (1.2) has at least two sign-changing solutions. Moreover, BVP (1.1), (1.2) has at least two positive solutions and two negative solutions.

Proof Since $G(t, s)$ is positive on $[2, T]_{\mathbb{Z}} \times[1, T]_{\mathbb{Z}}$, by $(\mathrm{H} 5)$, we have for any $u \in E$ with $\|u\|=M$,

$$
\begin{aligned}
|A u(t)| & =\left|\sum_{s=2}^{T} \sum_{k=2}^{T} G_{2}(t, k) G_{1}(k, s) f(t, u(t))\right| \leq \sum_{s=2}^{T} \sum_{k=2}^{T} G_{2}(t, k) G_{1}(k, s)|f(t, u(t))| \\
& <\omega^{-1} M \cdot \sum_{s=2}^{T} \sum_{k=2}^{T} G_{2}(t, k) G_{1}(k, s) \leq M, \quad \forall t \in[2, T]_{\mathbb{Z}} .
\end{aligned}
$$

This gives

$$
\|A u\|<M=\|u\| .
$$

By (5.2) and Lemmas 2.2 and 2.3, we have

$$
\begin{aligned}
& \operatorname{deg}(I-A, B(\theta, M), \theta)=1, \\
& i(A, P \cap B(\theta, M), P)=1, \\
& i(A,-P \cap B(\theta, M), P)=1 .
\end{aligned}
$$


From (H3) and Lemma 3.1, one has that the eigenvalues of the operator $A^{\prime}(\theta)=\beta_{0} K$ which are larger than 1 are

$$
\beta_{0} \lambda_{0}^{2}, \beta_{0} \lambda_{1}^{2}, \ldots, \beta_{0} \lambda_{k_{0}}^{2}
$$

From (H4) and Lemma 3.1, one has that the eigenvalues of the operator $A^{\prime}(\infty)=\beta_{\infty} K$ which are larger than 1 are

$$
\beta_{\infty} \lambda_{0}^{1}, \beta_{\infty} \lambda_{1}^{2}, \ldots, \beta_{\infty} \lambda_{k_{1}}^{2}
$$

It follows from Lemmas 2.4 and 2.5 that there exist $0<r_{1}<r_{0}$ and $R_{1}>R_{0}$ such that

$$
\begin{aligned}
& \operatorname{deg}\left(I-A, B\left(\theta, r_{1}\right), \theta\right)=(-1)^{k_{0}}=1, \\
& \operatorname{deg}\left(I-A, B\left(\theta, R_{1}\right), \theta\right)=(-1)^{k_{1}}=1,
\end{aligned}
$$

where $r_{0}$ and $R_{0}$ are given in Lemma 4.5. Owing to Lemma 4.3, one has

$$
\begin{aligned}
& i\left(A, P \cap B\left(\theta, r_{1}\right), P\right)=0, \\
& i\left(A,-P \cap B\left(\theta, r_{1}\right),-P\right)=0, \\
& i\left(A, P \cap B\left(\theta, R_{1}\right), P\right)=0, \\
& i\left(A,-P \cap B\left(\theta, R_{1}\right),-P\right)=0 .
\end{aligned}
$$

According to the additivity of the fixed point index, by (5.4), (5.10), and (5.12), we have

$$
\begin{aligned}
i\left(A, P \cap\left(B(\theta, M) \backslash \overline{B\left(\theta, r_{1}\right)}\right), P\right) & =i(A, P \cap B(\theta, M), P)-i\left(A, P \cap B\left(\theta, r_{1}\right), P\right) \\
& =1-0=1, \\
i\left(A, P \cap\left(B\left(\theta, R_{1}\right) \backslash \overline{B(\theta, M)}\right), P\right) & =i\left(A, P \cap B\left(\theta, R_{1}\right), P\right)-i(A, P \cap B(\theta, M), P) \\
& =0-1=-1 .
\end{aligned}
$$

Hence, the nonlinear operator $A$ has at least two fixed points $u_{1} \in P \cap\left(B(\theta, M) \backslash \overline{B\left(\theta, r_{1}\right)}\right)$ and $u_{2} \in P \cap\left(B\left(\theta, R_{1}\right) \backslash \overline{B(\theta, M)}\right)$, respectively. Then, $u_{1}$ and $u_{2}$ are positive solutions of BVP (1.1), (1.2). Using again the additivity of the fixed point index, by (5.5), (5.11), and (5.13), we get

$$
\begin{aligned}
& i\left(A,-P \cap\left(B(\theta, M) \backslash \overline{B\left(\theta, r_{1}\right)}\right),-P\right)=1-0=1, \\
& i\left(A,-P \cap\left(B\left(\theta, R_{1}\right) \backslash \overline{B(\theta, M))},-P\right)=0-1=-1 .\right.
\end{aligned}
$$

Hence, the nonlinear operator $A$ has at least two fixed points $u_{3} \in-P \cap\left(B(\theta, M) \backslash \overline{B\left(\theta, r_{1}\right)}\right)$ and $u_{4} \in-P \cap\left(B\left(\theta, R_{1}\right) \backslash \overline{B(\theta, M)}\right)$, respectively. Then, $u_{3}$ and $u_{4}$ are negative solutions of $\operatorname{BVP}(1.1),(1.2)$.

Let

$$
\begin{aligned}
& \Gamma_{1}=\left\{u \in P \cap\left(B(\theta, M) \backslash \overline{B\left(\theta, r_{1}\right)}\right): A u=u\right\}, \\
& \Gamma_{2}=\left\{u \in P \cap\left(B\left(\theta, R_{1}\right) \backslash \overline{B(\theta, M)}\right): A u=u\right\},
\end{aligned}
$$




$$
\begin{aligned}
& \Gamma_{3}=\left\{u \in-P \cap\left(B(\theta, M) \backslash \overline{B\left(\theta, r_{1}\right)}\right): A u=u\right\}, \\
& \Gamma_{4}=\left\{u \in-P \cap\left(B\left(\theta, R_{1}\right) \backslash \overline{B(\theta, M)}\right): A u=u\right\} .
\end{aligned}
$$

It follows from Lemmas 2.6, 4.3, Remark 4.2, and (5.14)-(5.17) that there exist open subsets $\mathrm{O}_{1}, \mathrm{O}_{2}, \mathrm{O}_{3}$, and $\mathrm{O}_{4}$ of $E$ such that

$$
\begin{aligned}
& \Gamma_{1} \subset O_{1} \subset P \cap\left(B(\theta, M) \backslash \overline{B\left(\theta, r_{1}\right)}\right), \quad \Gamma_{1} \subset O_{1} \subset P \cap\left(B\left(\theta, R_{1}\right) \backslash \overline{B(\theta, M)}\right), \\
& \Gamma_{3} \subset O_{3} \subset-P \cap\left(B(\theta, M) \backslash \overline{B\left(\theta, r_{1}\right)}\right), \quad \Gamma_{4} \subset O_{4} \subset-P \cap\left(B\left(\theta, R_{1}\right) \backslash \overline{B(\theta, M)}\right), \\
& \operatorname{deg}\left(I-A, O_{1}, \theta\right)=1, \\
& \operatorname{deg}\left(I-A, O_{2}, \theta\right)=-1, \\
& \operatorname{deg}\left(I-A, O_{3}, \theta\right)=1, \\
& \operatorname{deg}\left(I-A, O_{4}, \theta\right)=-1 .
\end{aligned}
$$

By (5.3), (5.20), (5.22), (5.8), and the additivity of the Leray-Schauder degree, we get

$$
\operatorname{deg}\left(I-A, B(\theta, M) \backslash\left(\overline{O_{1}} \cup \overline{O_{3}} \cup \overline{B\left(\theta, r_{1}\right)}\right), \theta\right)=1-1-1-1=-2,
$$

which implies that the nonlinear operator $A$ has at least one fixed point $u_{5} \in B(\theta, M) \backslash\left(\overline{O_{1}} \cup\right.$ $\left.\overline{O_{3}} \cup \overline{B\left(\theta, r_{1}\right)}\right)$.

Similarly, by (5.9), (5.21), (5.23), and (5.3), we get

$$
\operatorname{deg}\left(I-A, B\left(\theta, R_{1}\right) \backslash\left(\overline{O_{2}} \cup \overline{O_{4}} \cup \overline{B(\theta, M)}\right), \theta\right)=1+1+1-1=2,
$$

which implies that the nonlinear operator $A$ has at least one fixed point $u_{6} \in B\left(\theta, R_{1}\right) \backslash\left(\overline{O_{2}} \cup\right.$ $\left.\overline{O_{4}} \cup \overline{B(\theta, M)}\right)$. Then, $u_{5}$ and $u_{6}$ are two distinct sign-changing solutions of BVP (1.1), (1.2). Thus, the proof of Theorem 5.1 is finished.

Theorem 5.2 Assume that the conditions (H1)-(H5) hold, and that $f(t, x)=-f(t,-x)$ for $t \in$ $[2, T]_{\mathbb{Z}}$ and $x \in \mathbb{R}$. Then $B V P(1.1),(1.2)$ has at least four sign-changing solutions. Moreover, $B V P$ (1.1), (1.2) has at least two positive solutions and two negative solutions.

Proof It follows from the proof of Theorem 5.1 that BVP (1.1), (1.2) has at least six different nontrivial solutions $u_{i}(i=1,2, \ldots, 6)$ satisfying

$$
\begin{aligned}
& u_{1}, u_{2} \in P^{\circ}, \quad u_{3}, u_{4} \in-P^{\circ}, \quad u_{5}, u_{6} \notin P \cup(-P), \\
& r_{1}<\left\|u_{5}\right\|<|M|<\left\|u_{6}\right\|<R_{1} .
\end{aligned}
$$

By the condition that $f(t, x)=-f(t,-x)$ for $t \in[2, T]_{\mathbb{Z}}$ and $x \in \mathbb{R}$, we know that $-u_{5}$ and $-u_{6}$ are also solutions of BVP (1.1), (1.2). Let $u_{7}=-u_{5}, u_{8}=-u_{6}$, then $u_{i}(i=1,2, \ldots, 8)$ are different nontrivial solutions of BVP (1.1), (1.2). The proof is completed.

By the method used in the proof of Theorems 5.1 and 5.2, we can prove the following corollaries. 
Corollary 5.3 Assume that the conditions (H1)-(H3) and (H5) or (H1), (H2), (H4), and (H5) hold. Then BVP (1.1), (1.2) has at least one sign-changing solution. Moreover, BVP (1.1), (1.2) has at least one positive solution and one negative solution.

Corollary 5.4 Assume that the conditions (H1)-(H3) and (H5) or (H1), (H2), (H4), and (H5) hold, and that $f(t, x)=-f(t,-x)$ for $t \in[2, T]_{\mathbb{Z}}$ and $x \in \mathbb{R}$. Then BVP (1.1), (1.2) has at least two sign-changing solutions. Moreover, BVP (1.1), (1.2) has at least one positive solution and one negative solution.

\section{Competing interests}

The author declares that they have no competing interests.

\section{Acknowledgements}

The author is very grateful to the anonymous referees for their valuable suggestions.

Received: 20 October 2012 Accepted: 27 December 2012 Published: 14 January 2013

\section{References}

1. Agarwal, RP: Difference Equations and Inequalities: Theory, Methods, and Applications, 2nd edn. Dekker, New York (2000)

2. Agarwal, RP, O'Regan, D, Wong, PJY: Positive Solutions of Differential, Difference and Integral Equations. Kluwer Academic, Dordrecht (1999)

3. Gao, CH, Dai, GW, Ma, RY: Existence of positive solutions to discrete second-order boundary value problems with indefinite weight. Adv. Differ. Equ. 2012, 3 (2012). 39A12 (34B18 39A23)

4. Wong, PJY, Xie, L: Three symmetric solutions of Lidstone boundary value problems for difference and partial difference equations. Comput. Math. Appl. 45, 1445-1460 (2003)

5. Yu, JS, Guo, ZM: On boundary value problems for a discrete generalized Emden-Fowler equation. J. Differ. Equ. 231, 18-31 (2006)

6. Yang, Y, Zhang, JH: Infinitely many mountain pass solutions on a kind of fourth-order Neumann boundary value problem. Appl. Math. Comput. 213, 262-271 (2009)

7. Yang, Y, Zhang, JH: Nontrivial solutions on a kind of fourth-order Neumann boundary value problems. Appl. Math. Comput. 218, 7100-7108 (2012)

8. He, TS, Yang, W, Yang, FJ: Sign-changing solutions for discrete second-order three-point boundary value problems. Discrete Dyn. Nat. Soc. 2010, Article ID 705387 (2010). doi:10.1155/2010/705387

9. Guo, DJ, Sun, JX, Liu, ZL: Functional Methods for Nonlinear Differential Equations. Shandong Science and Technology Press, Jinan (1995)

10. Guo, DJ, Lakshmikantham, V: Nonlinear Problems in Abstract Cones. Notes and Reports in Mathematics in Science and Engineering, vol. 5. Academic Press, Boston (1988)

11. Guo, DJ: Nonlinear Functional Analysis, 2nd edn. Shandong Science and Technology Press, Jinan (2001)

12. Krasnoselskiĭ, MA, Zabre ǩk, PP: Geometrical Methods of Nonlinear Analysis. Grundlehren der Mathematischen Wissenschaften, vol. 263. Springer, Berlin (1984)

13. Agarwal, RP, Bohner, M, Wong, PJY: Sturm-Liouville eigenvalue problems on time scales. Appl. Math. Comput. 99, 153-166 (1999)

doi:10.1186/1687-1847-2013-10

Cite this article as: Yang: Sign-changing solutions to discrete fourth-order Neumann boundary value problems. Advances in Difference Equations 2013 2013:10

\section{Submit your manuscript to a SpringerOpen ${ }^{\circ}$ journal and benefit from:}

- Convenient online submission

- Rigorous peer review

- Immediate publication on acceptance

- Open access: articles freely available online

- High visibility within the field

- Retaining the copyright to your article 